\title{
Articulación De Arquetipos Organizacionales Con Base A Raúl Espejo Y Peter Senge
}

Maldonado Méndez Juan Sebastián , Suárez Russi Yensi Marianne, Vargas Medina Libet Nathalia

Universidad Autónoma De Colombia

Recibido: 15/04/2021 Revisado: 21/05/2021 Aceptado: 26/06/2021 Publicado: 28/07/2021

\section{Resumen}

En el siguiente texto se explica la estrecha relación que existe entre los cuatro elementos de la quinta disciplina de Peter Senge y los cuatro aspectos de Raúl Espejo para formar una organización recursiva. Para complementar esta relación, se identifican los elementos (dominio personal, modelos mentales, visión compartida y aprendizaje en equipo) y aspectos (identidad, cohesión, ciudadanía y desempeño) dentro de los arquetipos organizacionales y cómo estos sirven de herramienta para establecerlos.

\section{Palabras Clave}

Identidad, cohesión, ciudadanía, desempeño, dominio personal, modelos mentales, visión compartida, aprendizaje en equipo.

\begin{abstract}
The following text explains the close relationship that exists between the four elements of Peter Senge's fifth discipline and the four aspects of Raúl Espejo to form a recursive organization. To complement this relationship, the elements (personal domain, mental models, shared vision and team learning) and aspects (identity, cohesion, citizenship and performance) within the organizational archetypes and how they serve as a tool to establish them are identified.
\end{abstract}

BIOTECH \& ENGINEERING Untels. Jul - Dic.1(2), 2021; ISSN:2788 -4295; 62-74 DOI: https://doi.org/10.52248/eb.Vol1Iss02.23 


\section{Keywords}

Identity, cohesion, citizenship, performance, personal mastery, mental models, shared vision, team learning.

\section{Introducción}

En este informe se presenta el resultado de la investigación sobre arquetipos organizacionales realizada en el Semillero HSEQ. De acuerdo con la profundización, análisis del tema y fuentes consultadas, se desarrolla en primera instancia que los arquetipos son las actitudes, comportamientos y conductas que tiene un individuo, grupo o sociedad en conjunto frente a diversas situaciones o contextos que con el tiempo se representan de forma automática y continua. En segunda instancia y focalizándose en el objetivo de la investigación abarca los arquetipos organizacionales que hacen referencia al comportamiento de los individuos, grupos o sociedades en conjunto dentro de una organización que se caracterizan por tener un pensamiento sistémico.

\section{Aspectos teóricos}

\subsection{La quinta disciplina}

"Las organizaciones inteligentes son posibles porque en el fondo todos somos aprendices"

En la quinta disciplina (Senge, 2010) menciona la importancia del aprendizaje en equipo dentro de las organizaciones. La forma de aprendizaje también se debe aprender al igual que aprender en grupo, es por esto que se comienza la reflexión de la quinta disciplina con la frase inicialmente plasmada.

Las organizaciones deben estar en constante aprendizaje, por lo que es importante tener claro el concepto de pensamiento sistémico, que hace referencia a los comportamientos que generan actos interrelacionados entre los individuos, grupos, equipos y elementos que conforman la organización. Para el autor, las empresas deben brindar experiencias a sus trabajadores que vayan más allá de 
cubrir sus necesidades, esto hace referencia a que las tareas contribuyen al logro de los resultados. Teniendo en cuenta lo anterior, se resalta la importancia del buen comportamiento de los individuos en pro del buen funcionamiento de la organización.

La disciplina de la organización inteligente tiene como fundamento la innovación que se presenta cuando se desarrolla una idea, en donde se desprenden aspectos como la tecnología, el uso eficiente de los recursos existentes, el beneficio característico de la misma. Además, una idea innovadora puede desarrollarse con base a ideas procedentes del tema en cuestión.

Explicando las cinco disciplinas, las personas juegan el papel más importante en cada una de ellas, por lo que es indispensable hablar del rol que cumplen los individuos dentro de una organización inteligente. Se menciona el Dominio personal, el cual explica la importancia de cómo influye la vida personal en la vida laboral, por lo que se habla del desarrollo de la paciencia, la concentración de la energía y el ver la realidad desde un punto objetivo ya que es gracias a las personas que la organización desarrolla con normalidad sus funciones cotidianas, lo que nos lleva a la idea que la organización debe priorizar el bienestar del capital humano mediante un aprendizaje donde el empleado y la organización encuentren beneficio personal y laboral pues se debe tener en cuenta que lo bueno y lo malo es el resultado de las diferentes decisiones, actitudes y comportamientos que conforman el ente corporativo.

Los modelos mentales hacen referencia a cómo la planificación empresarial se convierte en aprendizaje institucional, lo que hace tener una lógica de la realidad que está rodeado el empleado dentro del ambiente laboral, lo que conlleva a que el capital humano de la organización adquiera una serie de aptitudes con el fin impartir una visión compartida. La relación de los individuos en el ambiente laboral lleva a que exista un aprendizaje en equipo, lo que maximizará los resultados, pues esto genera diálogo dentro del equipo lo que significa alcanzar percepciones que no se alcanzan individualmente y que al mismo tiempo elimina supuestos individuales, esto quiere decir si el equipo aprende la organización aprende. Las grandes organizaciones no se construyen tratando de imitar a otras, así como la grandeza individual no se logra tratando de copiar a otra "gran persona". 
La quinta disciplina se basa en el pensamiento sistémico, la cual unifica las cuatro disciplinas mencionadas anteriormente, dominio personal, modelos mentales, construcción de una visión compartida y aprendizaje en equipo que combina la práctica y teoría haciendo que los resultados de cada una repercutan en las demás, es por esto que el pensamiento sistémico juega el papel más importante dentro de una organización inteligente

\subsection{Organizaciones recursivas}

Este tipo de organización es aquella que desarrolla una idea de una unidad autónoma dentro de otra unidad autónoma, lo que hace referencia a un pensamiento sistémico organizacional como fundamento de una organización recursiva, para este caso las unidades hacen se refieren a las personas que conforman al capital humano, estas unidades requieren de un fortalecimiento dividido en cuatro aspectos, identidad, cohesión, ciudadanía y desempeño, explicándoles a continuación.

\subsubsection{Identidad}

La identidad es un aspecto clave de las organizaciones recursivas debido a que cada actividad debe tener sus propios propósitos de identidad; si no es así cambia el enfoque a una organización jerárquica y no recursiva. El problema de identidad es realmente significativo en el momento en que solo se crean hipótesis dentro de la organización e impide que funcione como un todo. Por tal motivo no ser puede ser autónomo si no se crean los propios fines o no se genera responsabilidad de la identidad

\subsubsection{Cohesión}

La cohesión es un requisito para la producción de las actividades primarias en una organización. Cuando las empresas gastan muchos recursos para lograr la cohesión interna, menos recursos se centran en producir las tareas propias que deben ejercer dentro de la organización, es decir que se llega a un punto en que los cotos de lograr la cohesión exceden sus beneficios por tal motivo es vital centrarse y dar importancia a la comunicación interna. 


\subsubsection{Ciudadanía}

Parte del hecho de que se pertenece a una organización, lo que conlleva a un esfuerzo y atención a todos los lineamientos de comportamiento que lo componen, esto no quiere decir que los lineamientos de la misma van en contra a quien pertenecen, sino que se lleva un respeto mutuo del uno y otro.

\subsubsection{Desempeño}

Este aspecto hace referencia a la relación del desempeño con las actividades primarias de la organización que tiene una estrecha relación con cada una de las personas que tienen a cargo esta función, estas funciones parten de un individuo o equipo el cual se caracteriza por tener una cadena de valor, esta actividad tendrá relación con proveedores de bienes o servicios por lo que la relación con ellos debe de ser la más acertada pues estos proveedores deben de estar alienados con aquellas actividades primarias las cuales tienen a cargo, esto significa que el desempeño se verá reflejado en la adaptación al cambio de las condiciones internas y externa.

Según lo expuesto (Espejo, 1999) sobre las organizaciones recursivas, se deben de identificar cuatro aspectos, identidad, cohesión, ciudadanía y desempeño las cuales se deben reforzar continuamente, estos aspectos se presentan en los diferentes individuos que conforman la organización y quienes son los encargados de garantizar el cumplimiento de los objetivos propuestos los cuales se logran con la unión de los cuatro aspectos anteriormente mencionados.

\section{Desarrollo}

Durante las sesiones del grupo del semillero de investigación y con el apoyo de los docentes ingenieros, se socializan las normas de gestión de calidad como la ISO 9000. ISO 14000, ISO 45000; modelos de gestión de calidad, obstáculos de aprendizaje y entre otras. Estos temas se han tratado con el fin de ampliar el contexto en el cual se puede aplicar la teoría dentro del ambiente organizacional. Adicionalmente, se realiza una serie de comparaciones entre las ideas propuestas 
por Peter Senge con las cinco disciplinas y Raúl espejos con los cuatro aspectos de una organización recursiva en relación a los arquetipos.

\subsection{Arquetipos organizacionales según Raúl Espejo}

\subsubsection{Identidad.}

a) El empleado no sigue el conducto regular que ha sido estipulado desde un principio con el fin de cumplir una tarea y/o función. El conducto regular es el medio por el cual se transmiten las órdenes, solicitudes, informes y reclamaciones dentro de una empresa. Esto con el fin de tener un control y un manejo adecuado de diversas situaciones que puedan generar inconvenientes dentro de la organización, al no cumplirlo se puede ver afectado el desempeño, la comunicación, la motivación y el logro de objetivos en el tiempo planeado

b) Un miembro del equipo desea asumir un rol que no le corresponde, lo que conlleva a un mal ambiente laboral. Actualmente los trabajadores deben tener la capacidad de llevar a cabo cualquier tarea que se le asigne dentro de la empresa y que esté dentro de su perfil y capacidades. Sin embargo, el no tener claras sus funciones genera varios inconvenientes tanto para quien lo está haciendo como para los demás. Al estar disperso o querer realizar tareas que no corresponden dificultará que el empleado pueda enfocarse y trabajar en torno a las metas establecidas, se genera un mal ambiente laboral ya que baja la productividad, se tienen actitudes negativas entre compañeros, entre otros. Es importante tener una descripción del cargo, buen proceso de reclutamiento, programas de capacitación pertinentes, evaluar carga de trabajo.

c) Un empleado, a pesar de haber tenido la inducción empresarial, no es capaz de definir cuál es la misión y objetivos de la empresa, o el impacto que tiene su rol dentro de la misma. Es importante que los integrantes de una organización tengan muy claro primero cual es la misión y cuáles son los objetivos a los que quiere llegar la empresa; segundo saber el 
impacto o importancia que tienen sus funciones para el desarrollo y cumplimiento de dichos objetivos ya que esto es esencial para crear un compromiso, realizar las acciones necesarias y enfocarse hacia lo que se quiere lograr.

d) Un empleado tiene comportamientos no deseados dentro de su ambiente laboral. Una conducta incorrecta o mal comportamiento de uno o varios empleados dentro de una organización no solo afecta el ambiente laboral, sino que perturba el buen desarrollo de las actividades. Esto se puede presentar por la presión laboral, falta de comunicación o factores externos como problemas personales.

\subsubsection{Cohesión:}

(Espejo, 1999) La cohesión hace referencia al buen relacionamiento interno de los diferentes sistemas, áreas, personas etc, que buscan cumplir los objetivos de la organización, esto se logra mediante las diferentes actividades del ambiente laboral, el control, los procesos ágiles y acertados, el enlace de todas las funciones que tienen a cargo los diferentes asociados a la organización. Para este aspecto se ha identificado diferentes arquetipos que afectan directamente la cohesión y por ende el cumplimiento de los objetivos de la organización.

a) No existe comunicación asertiva entre las diferentes relaciones que hay en la organización:

Este caso se presenta en la mayoría de los ambientes laborales, desde el equipo de trabajo, pasando por el área y llegando hasta la alta dirección. La comunicación es un elemento esencial para que haya una cohesión a nivel interno pues es así que todos los involucrados tendrán conocimiento de que se hizo, que se está haciendo y que se hará.

b) Falta de coordinación entre áreas: Las áreas que conforman la organización tienen un fin específico que aporta en gran medida a los objetivos que se plantea el corporativo, la relación de las diferentes áreas debe de ser lo más estrecha posible ya que pertenecen a un sistema que permite recoger información para toma de decisiones, es allí donde la cohesión debe de ser acertada.

BIOTECH \& ENGINEERING Untels. Jul - Dic.1(2), 2021; ISSN:2788 -4295; 62-74 
c) Existe mal manejo de la información en las diferentes áreas: Este arquetipo identificado va muy de la mano al anterior, la información que se maneja debe de ser legible, confiable y acertada, dado que de esta surgirán decisiones y acciones que se espera aporten de forma positiva a los objetivos de la organización.

d) No se realizan las actividades en un orden lógico y/o coherente: El orden en el tiempo es uno de los factores que influyen en la cohesión, es allí donde tienen que coordinar las áreas, equipos de trabajos y diferentes sistemas dentro del ambiente laboral que se realicen y se cumplan con las funciones asignadas en pro del resultado que se espera.

\subsubsection{Desempeño}

a) Las malas relaciones interpersonales afectan el ambiente laboral causando que no se consigan los resultados esperados: Para los seres sociales, no es un secreto que la productividad se puede ver afectada por las relaciones que existen con otros individuos dentro de la organización; Por ende, es importante crear espacios seguros en donde los empleados puedan manifestar a Recursos Humanos todos aquellos problemas que se presentan con compañeros.

b) La responsabilidad asignada al empleado: Es importante que los empleados sean lo suficientemente conscientes de la importancia que tiene su rol dentro de la organización, deben estar dispuestos a dar lo mejor de sí mismos, ya que no tomarse en serio su rol, afectará el desempeño y estos se puede reflejar en los resultados tanto individuales como colectivos.

c) La perturbación del ambiente laboral afecta el desempeño: Como se mencionó en el literal a de esta sección, existen otros factores que pueden repercutir de forma negativa, tales como el método de trabajo que usa el equipo, sistema, área etc., la forma en que emplean los recursos asignados para determinada tarea.

BIOTECH \& ENGINEERING Untels. Jul - Dic.1(2), 2021; ISSN:2788 -4295; 62-74 


\subsubsection{Ciudadanía}

a) Las malas relaciones interpersonales hacen que se pierda la armonía en el ambiente laboral: No tener buenas relaciones interpersonales pueden desencadenar una serie de problemas tanto para los individuos, áreas y la propia organización, por lo que es importante que los empleados también tengan inteligencia emocional y/o trabajen en ella, que no mezclen problemas laborales con personales o de otra índole, que siempre estén abiertos al diálogo y a las críticas constructivas.

b) No seguir el manual de convivencia de la empresa: Estos documentos son diseñados con el fin de crear un ambiente sano para sus empleados, el no acatarlos implica que los empleados no tienen respeto ni por la organización, ni por sus compañeros de trabajo, ya que puede llegar a violentar la sana convivencia o incluso su propio bienestar.

c) No hacer buen uso de los recursos asignados por la organización: Como buenos ciudadanos (empleados), entre nuestras obligaciones debe estar el velar por el buen uso de los recursos de la organización. El realizar acciones que vayan en contra de esto, puede demostrar ignorancia, pero en otros demuestra que se ejerce un rol errado frente a la organización.

d) No tener sentido de pertenencia frente al rol que se ha asignado: Es importante que los empleados se apropien no sólo de su rol, sino también de todas las circunstancias que rodean dicho rol y/o son conscientes de ello.

\section{Relación entre arquetipos}




\begin{tabular}{|l|l|l|l|l|}
\hline ARQUETIPO & IDENTIDAD & COHESIÓN & DESEMPEÑO & CIUDADANIA \\
\hline $\begin{array}{l}\text { Soluciones } \\
\text { contraproducentes }\end{array}$ & $\mathrm{X}$ & $\mathrm{X}$ & \\
\hline $\begin{array}{l}\text { Límites de } \\
\text { crecimiento }\end{array}$ & & $\mathrm{X}$ & $\mathrm{X}$ \\
\hline $\begin{array}{l}\text { Tragedia del } \\
\text { terreno común }\end{array}$ & & $\mathrm{X}$ & $\mathrm{X}$ \\
\hline $\begin{array}{l}\text { Desplazamiento } \\
\text { de la carga }\end{array}$ & & $\mathrm{x}$ & $\mathrm{X}$ & \\
\hline $\begin{array}{l}\text { Adversarios } \\
\text { accidentales }\end{array}$ & & & & \\
\hline $\begin{array}{l}\text { Éxito para quien } \\
\text { tiene éxito }\end{array}$ & & & & \\
\hline
\end{tabular}

Por otro lado, la quinta disciplina además de relacionarse es la unión de los cuatro (4) aspectos, siendo el dominio personal, los modelos mentales, la construcción de una visión compartida y el aprendizaje en equipo.

$\mathrm{Al}$ hablar de la identidad como un aspecto se tiene en cuenta el fin que tiene el individuo dentro de la organización, que al mismo tiempo muestra quien es en realidad, lo que se relaciona con el dominio personal, lo que conlleva sacar mayor provecho de los recursos. Continuando, la cohesión indica que la relación y el comportamiento entre los individuos, grupos, equipos, áreas 
y demás unidades, logra aumentar los beneficios, y mediante este se relacionan los modelos mentales de la quinta disciplina, pues para que esto suceda se debe realizar una planificación corporativa lo que lleva a un aprendizaje institucional

La construcción de la una visión compartida hace referencia a que los individuos sean conscientes que pertenecen a una organización y logra que cada uno de ellos comparta la idea impuesta por los entes superiores, si llevamos esta idea al escenario del aspecto de ciudadanía se puede decir que tienen una relación estrecha pues es ciudadano quien respeta los lineamientos del otro, pero al mismo tiempo tienen alineamientos en común.

El aprendizaje en equipo ofrece una serie de beneficios los cuales contribuyen en gran medida al cumplimiento de resultados, de estos resultan el aspecto de desempeño, pues mediante este se logra medir en qué cantidad contribuye la unidad en los objetivos propuestos.

\section{Resultados}

Establecer comportamientos de los individuos objeto para poder identificar si existen aspectos o elementos planteados por Raúl Espejo y Peter Senge, y definir si existen arquetipos organizacionales.

La Herramienta para gestión de arquetipos organizacionales, es una matriz que sirve para establecer, gestionar y mitigar los arquetipos organizacionales mediante la descripción de comportamientos o actitudes que presentan los individuos y cómo afecta los procesos de la organización. Para esto, se sitúan dichos comportamientos o actitudes en el aspecto o elemento que se ve afectado y así, lograr establecer el arquetipo sobre el cual se debe trabajar, posterior a ello se proponen acciones para mitigar el impacto del mismo. Con el fin de dejar claro el funcionamiento de "la Herramienta para gestión de arquetipos organizacionales" se presenta un ejemplo práctico. 

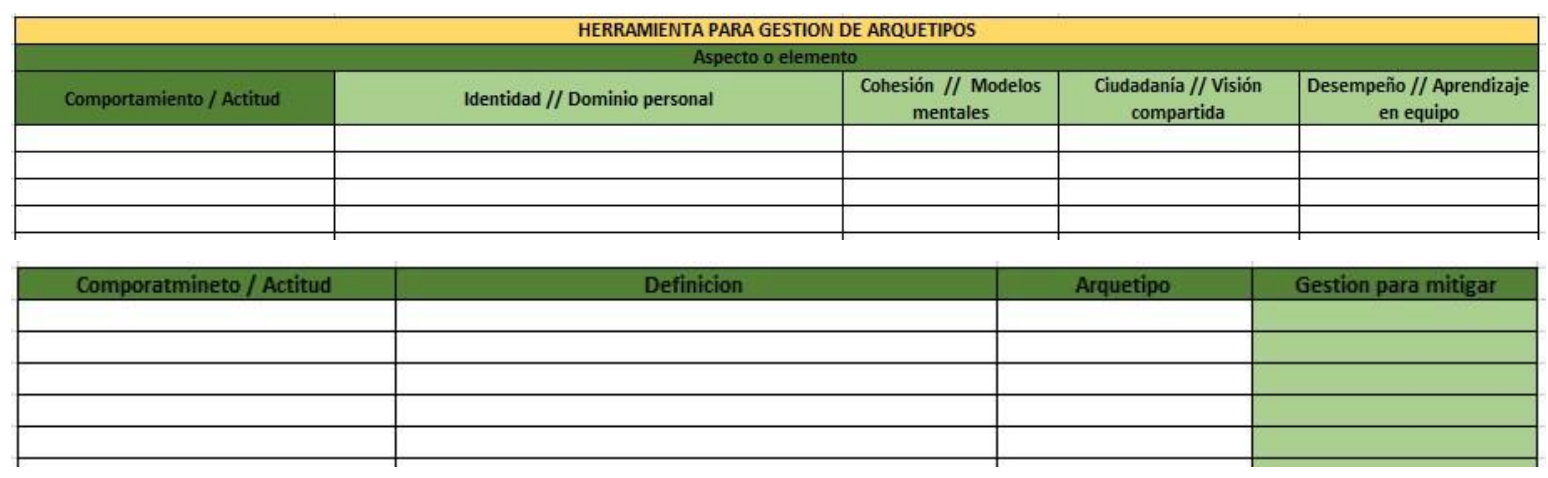

\section{Instrucciones}

1. Identificar el comportamiento o actitud que considere generan ruido en los procesos de la organización.

2. Ubicar el comportamiento o actitud dentro de los aspectos y elementos.

\begin{tabular}{|c|c|c|c|c|}
\hline \multicolumn{5}{|c|}{ HERRAMIENTA PARA GESTION DE ARQUETTPOS } \\
\hline \multicolumn{5}{|c|}{ Aspecto o elemento } \\
\hline Comportamiento / Actitud & Identidad // Dominio personal & $\begin{array}{c}\text { Cohesión // Modelos } \\
\text { mentales }\end{array}$ & $\begin{array}{c}\text { Ciudadania // Visión } \\
\text { compartida }\end{array}$ & $\begin{array}{c}\text { Desempeio // Aprendizaje } \\
\text { en equipo }\end{array}$ \\
\hline $\begin{array}{l}\text { las personas de compan̂ía } \\
\text { utilizan mucho el celular }\end{array}$ & & & X & $x$ \\
\hline $\begin{array}{l}\text { los empleados gastas mucho } \\
\text { tiempo en el café }\end{array}$ & & & & $x$ \\
\hline
\end{tabular}

3. Definir cómo estos aspectos se encuentran dentro de los comportamientos o actitudes identificadas.

4. Establecer arquetipos.

5. Plantear medidas para mitigar el impacto del arquetipo. 


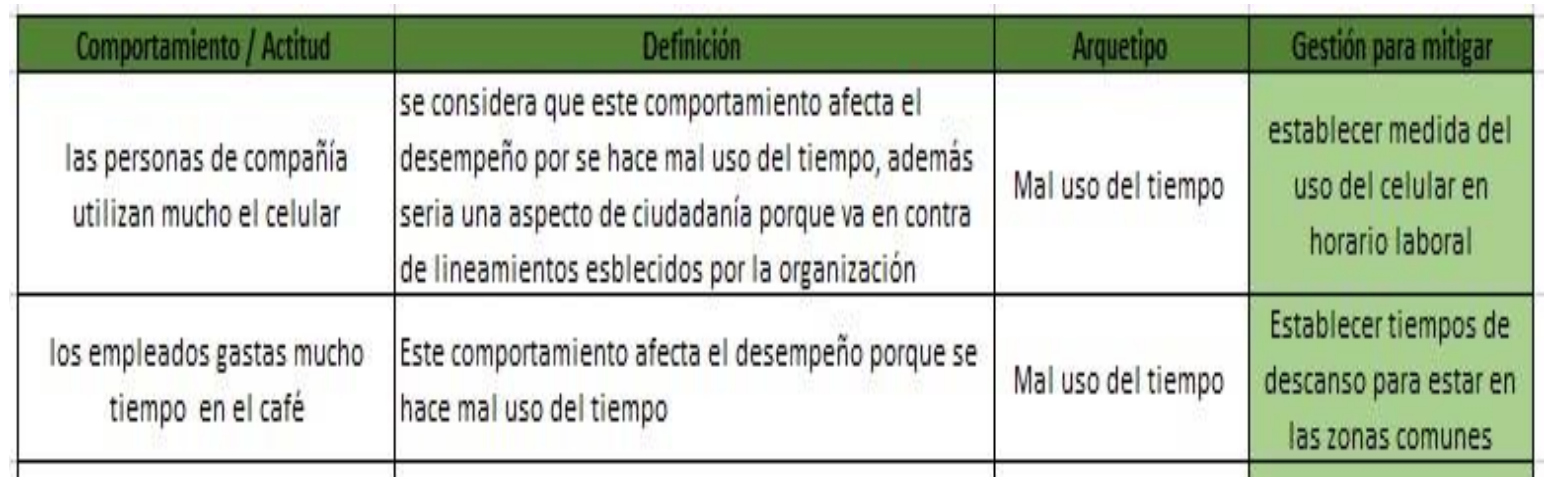

\section{Conclusión}

De acuerdo con la investigación realizada sobre los arquetipos organizacionales, se concluye que es necesario identificar y relacionar comportamientos, actitudes y conductas de los individuos dentro del ambiente laboral de una organización con los aspectos y los elementos de los autores en cuestión. Por otro lado, identificamos que existe una estrecha relación y un complemento entre los cuatro elementos de la quinta disciplina por Peter Senge y los cuatro aspectos de una organización recursiva por Raúl Espejo y que estos contribuyen a la mitigación del efecto negativo que ocasionan los arquetipos organizacionales en los procesos.

\section{Recomendación}

Antes de aplicar la herramienta, se sugiere realizar una contextualización de la empresa, ya que cada organización presenta dinámicas únicas que deben identificar y no sólo usar generalizaciones. Tener en cuenta diferentes aspectos internos y externos de la organización objetivo como el sector económico, la cultura organizacional, el organigrama, etc. Los resultados de la aplicación de la Herramienta dependen de la organización e individuos objeto.

\section{Bibliografía}

1. Andrés Mejía D. Gloria Maritza Díaz. Tipos de arcos y hacia dónde disparan: Sobre la naturaleza y posibilidades de los arquetipos. 15-06-2020, de Universidad de Los Andes 
2. Gonzalo Carlos Pérez Gómez. (2008)¿Cómo combinar la identificación de arquetipos organizacionales con la observación de obstáculos de aprendizaje organizacional para proponer ajustes estructurales en una entidad? Bogotá: Universidad de los Andes.

3. Walter Verlag A. G Olten. (2003). Arquetipos e inconsciente colectivo. MÉXICO: PAIDOS.

4. Peter Senge. (1990). La quinta disciplina. 2010: Ediciones Granica SA.

5. Andrés Mejía D. / Gloria Maritza Díaz. (NA). Tipos de arcos y hacia dónde disparan: Sobre la naturaleza y posibilidades de los arquetipos. En Bogotá, Colombia: Universidad de los Andes.

6. Espejo, R. (1999, agosto). Aspects of identity, cohesion, citizenship and performance in recursive organisations. University of Lincolnshire and Humberside.https://www.emerald.com/insight/content/doi/10.1108/03684929910282935/ $\underline{\text { full/html }}$ 